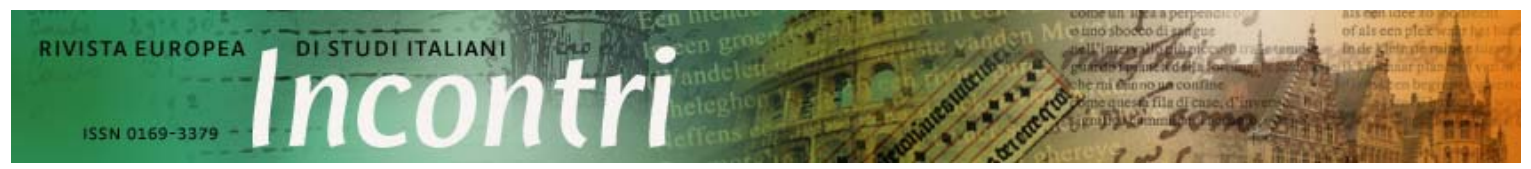

URN:NBN:NL:Ul:10-1-114246 - Publisher: Igitur publishing

Content is licensed under a Creative Commons Attribution 3.0 License

Anno 28, 2013 / Fascicolo 1 - Website: www.rivista-incontri.nl

\title{
La presenza subalterna in Italia e la scrittura come terapia
}

\section{Dagmar Reichardt}

\section{II subalterno}

Ricorrendo al mito platonico della caverna (in francese: antre) e all'idea dello spazio intermedio di un third space o in-between-space come lo avrebbe descritto Homi K. Bhabha nel 1994, ${ }^{1}$ Jacques Derrida ha coniato l'omonimo antre-entre per definire un'identità attraverso l'idea della differenza. ${ }^{2}$ Riprendendo questo approccio $e$ collegandolo sul piano argomentativo con le teorie di Michel Foucault e Ranajit Guha, Gayatri C. Spivak cerca di elaborare il concetto del soggetto condizionato dal sistema egemonico e della classe subalterna ovvero del 'subalterno' di per sé nel suo famoso saggio Can the subaltern speak? (1988). ${ }^{3}$ Il suo testo si conclude con la riflessione su ciò che rimane indicibile, ${ }^{4}$ sulla sensazione tipicamente postcoloniale della perdita, ${ }^{5}$ e sulle donne, sul loro silenzio di fronte alla violenza imperialista e alla dominanza maschile. Nel contesto coloniale la donna subalterna non possiede né una storia né una voce, e viene discriminata e stigmatizzata con un radoppiamento traumatico: 'If, in the context of colonial production, the subaltern has no history and cannot speak, the subaltern as female is even more deeply in shadow...' 6

Questa frase finale del saggio, che implica una definizione del subalterno attraverso il non avere un passato storico e quello di non poter parlare, e di trovarsi dunque nell' 'ombra' della storia e/o della società, nella letteratura e cultura italiana riecheggia nell'idea dei 'vinti' originalmente verghiana e verista. ${ }^{7}$ La domanda retorica

\footnotetext{
${ }^{1}$ Cfr. il primo capitolo (e quelli seguenti) di: H. K. Bhabha, The location of culture, New York, Routledge, 1994.

${ }^{2}$ Cfr. J. Derrida, La Dissémination, Paris, Seuil, 1972, p. 261.

3 Il testo è apparso dapprima in Marxism and the interpretation of culture (1988, pp. 271-313) ed è riapparso in: G. C. Spivak, 'Can the subaltern speak?', in: Bill Ashcroft, Gareth Griffiths, Helen Tiffin (a cura di), The postcolonial studies reader, London/New York, Routledge, 1995, pp. 24-28, qui pp. 25-26. Per ulteriori saggi di Spivak sul subalterno, sul femminismo e su questioni di potere cfr. G. C. Spivak: The Spivak reader. Selected works of Gayatri Chakravorty Spivak, a cura di D. Landry e G. MacLean, London/New York, Routledge, 1996.

${ }^{4}$ Spivak, Can the subaltern speak?, cit., p. 28: 'the notion of what the work cannot say becomes important'.

5 'The postcolonial intellectuals learn that their privilege is their loss' (Ibidem).

${ }^{6}$ Ibidem.

${ }^{7}$ Per i rapporti teorici tra Gramsci, Spivak e le figure cruciali della letteratura verista, come lo sono i 'vinti' di Verga, cfr. D. Reichardt, 'Paradigma mundi? Die Geschichte des postkolonialen Siziliendiskurses zwischen literarischer Alterität und Identität', in: Dagmar Reichardt (a cura di), L'Europa che comincia e finisce: Ia Sicilia. Approcci transculturali alla letteratura siciliana. Beiträge zur transkulturellen Annäherung an die
} 
nel titolo del saggio Can the subaltern speak?, che figura tra $i$ testi fondatori del postcolonialismo, ricorda nel contesto italiano la classe subalterna dei contadini, semplici artigiani e braccianti stremati nel 'ciclo dei vinti' di Verga, il mondo degli 'offesi' di Vittorini, il popolino ritratto nel cinema neorealista o più recentemente la lettaratura italofona di migrazione come nuova corrente creativa prodotta da soggetti emarginati. Allo stesso tempo la mancanza di un passato storico e di una voce che si possa registrare chiaramente e fortemente sono due sintomi che rimandano alla Trauma Theory della scuola di Yale, e quindi ad un evento iniziatico caratterizzato dalla violenza - fisica o psichica che sia, motivata politicamente o sul piano sociale - una violenza che può risultare anche dal fatto di essere soggetti coloniali o postcoloniali come principalmente ha rivelato il saggio Orientalism (1978) di Edward Said. Secondo Piotr Sztompka ${ }^{8}$ anche la migrazione - emigrazione o immigrazione che sia - in una cosiddetta 'modernità liquida' come la nostra, produce degli outcasts e wasted lives, ${ }^{9}$ e quindi, afferma Sztompka, dei traumi che richiedono una terapia.

Ciò che Zygmunt Bauman esprime seguendo la stessa tradizione post-marxista della Spivak, negli ultimi vent'anni si riflette anche nella psichiatria e psicologia transculturale ${ }^{10}$ e nel cosiddetto expressive writing. ${ }^{11}$ Il caso di un trauma collettivo o culturale - indipendentemente se sia stato generato da una situazione coloniale 0 postcoloniale come sostiene Said, o se risulta dai fattori complessi che generano la migrazione in un mondo globalizzato come illustrano Sztompka e Bauman - esige una cura e quindi degli strumenti terapeutici. Come ha dimostrato il paradigma di Pennebaker, ${ }^{12}$ la writing therapy, che a partire dai tardi anni Ottanta suscita una crescente attenzione nell'ambito del trattamento psicoterapeutico e che su un piano interdisciplinare può fornire una soluzione attraverso la sublimazione catartica, creativa e salvifica.

\section{II caso italiano: violenza e postcolonialismo}

Literatur Siziliens. Contributions to a Transcultural Approach to Sicilian literature, Frankfurt am Main et al., Peter Lang, 2006, pp. 87-107, cfr. qui specialmente il riferimento alla letteratura e cultura siciliana a p. 100, dove sottolineo l'importanza della scrittura per opporrsi all'oblio, per credere nella forza memorialistica della narrazione e negli ideali degli strati sociali sottoprivilegiati come, appunto, lo sono $i$ 'vinti' del Verga, ma anche tutti gli autori subalterni e le scrittrici migranti che presenterò qui in seguito.

${ }^{8}$ P. Sztompka, 'The Trauma of Social Change. A Case of Postcommunist Societies', in: J. Alexander, R. Eyerman, B. Giesen N. J. Smelser, P. Sztompka, Cultural trauma and collective identity, Berkeley, University of California Press, 2004, p. 162 ss.; sintomatica anche la frase: 'Change is behind all triumphs of humankind, but it is also a source of trauma' (ivi, p. 195).

${ }^{9}$ Con la terminologia in corsivo mi riferisco a Z. Bauman che dopo Liquid modernity (Cambridge, Polity Press, 2000) ha pubblicato il titolo Wasted lives. Modernity and its outcasts (Cambridge, Polity Press, 2004).

${ }^{10}$ Moltissimi i titoli italiani apparsi nell'ultimo decennio nel settore medico-terapeutico in combinazione con l'analisi e la psicoterapia transculturale, soprattutto con riferimento migratorio e sociologico, p.es. M. Andolfi (a cura di), Famiglie immigrate e psicoterapia transculturale, Milano, Franco Angeli, $2004 ; \mathrm{A}$. Sanella, Salute transculturale. Percorsi socio-sanitari, Milano, Franco Angeli, 2010; Alfredo Ancora, La consulenza sistemica transculturale. Famiglie e culture, Milano, Franco Angeli, 2000.

${ }^{11}$ L'espressione è presa dal titolo del saggio di K. A. Balkie e K. Wilhelm intitolato 'Emotional and physical health benefits of expressive writing', in: Advances in psychiatric treatment, 11 (2005), pp. 338-346, http: / /apt.rcpsych.org/content/11/5/338. full (9 aprile 2012).

${ }^{12}$ Negli anni Ottanta il psicologo statunitense J. W. Pennebaker ha iniziato diversi studi e sperimenti con la scrittura espressiva provando che un setting di 3 a 5 sedute, durante le quali un gruppo di pazienti scriveva per 15-20 minuti un testo illustrando i loro sentimenti e pensieri più profondi nel contesto di un trauma, ha prodotto risultati nettamente benefici al livello fisico e mentale dei pazienti. Questo fenomeno è stato definito con la nozione di The Pennebaker paradigm (cfr. il sito di J.W. Pennebaker, http://homepage.psy.utexas.edu/homepage/Faculty/Pennebaker/Home2000/JWPhome.htm). 
È in questa cornice teorica che vorrei proporre una breve panoramica di alcuni scrittori della migrazione, con speciale attenzione per la letteratura di immigrazione italofona al femminile e per il genere del romanzo, con l'intento di indagare la funzione estetica e vivificante della letteratura postcoloniale. ${ }^{13}$ Prima di approfondire questo proposito ricordo le particolarità del caso italiano. Dalle pubblicazioni critiche di Armando Gnisci, che in diverse sue opere si è occupato della mondializzazione, della liminarità, della creolizzazione e dell'identità ibrida in età globalizzata, ${ }^{14}$ risulta che le opere degli autori di migrazione abbiano rivitalizzato la letteratura italofona. L'idea centrale di Gnisci è che le recenti scritture dell'immigrazione (Immigrant Writing) sul piano estetico manifestano una ridefinizione costruttiva generata dal contatto fra culture diverse producendone una nuova, quella transculturale.

Senza annullare la loro originaria appartenenza, le autrici e gli autori migranti scrivono in italiano, dando voce a personaggi che incarnano identità complesse e che spesso rimandano a elementi di autobiografismo e/o traumi risultanti dal fenomeno della migrazione e dai profondi mutamenti psicologici che ne derivano. All'inizio della prima fase della produzione letteraria a firma di migranti che si esprimono nella lingua del paese ospitante, ossia nel triennio 1990-1992, vengono pubblicati i tre romanzi iniziatici di Pap Khouma dal Senegal (lo, venditore di elefanti), Salah Methnani dalla Tunisia (Immigrato) e Mohamed Bouchane dal Marocco (Chiamatemi Alì), ${ }^{15}$ tutti quanti scritti in italiano a quattro mani e apparsi nel 1990 - 'anno a cui generalmente si fa risalire l'inizio della letteratura italiana della migrazione'. ${ }^{16}$

L'inizio di questo nuovo filone è segnato da un violento avvenimento politico, ossia dall'omicidio di Jerry Essan Masslo, un lavoratore stagionale dal Sudafrica ucciso il 24 agosto 1989 a Villa Literno nella provincia di Caserta. ${ }^{17}$ Lo scrittore prevalentemente francofono Tahar Ben Jelloun parte da questo caso nel suo racconto Villa Literno, scritto in italiano a quattro mani (assistito da Egi Volterrani) e pubblicato prima sul quotidiano Il Mattino di Napoli, poi nella sua antologia Dove lo Stato non c'è. ${ }^{18}$ Fin dall'inizio della letteratura dell'immigrazione in Italia si manifesta in maniera evidente quanto il trauma migratorio - per autoresponsabilità propria o per colpa altrui - provenga da un atto rischioso, a volte violento, autodistruttivo e/o potenzialmente letale. La violenza è causata o dal caso ( $p$ eresempio quello della nascita in un paese povero), o dallo scontro di culture (culture shock), o ancora dalla discriminazione, dalla xenofobia e dal

\footnotetext{
${ }^{13}$ Per analizzare il ruolo delle donne nell'emigrazione italiana si rinvia alla Storia dell'emigrazione italiana. Partenze, a cura di P. Bevilacqua, A. De Clementi, E. Franzina, Roma, Donzelli, 2001, Parte prima, cap. IX; Parte seconda, cap. II (p. 5) e cap. IV. Si verifichi inoltre il volume di H. Barolini (a cura di), The dream book: An anthology of writings by Italian American women, New York, Schocken Books, 1987; e, per l'approccio postcoloniale, lo studio critico di S. Ponzanesi, Paradoxes of postcolonial culture. Contemporary women writing of the Indian and Afro-Italian diaspora, Albany, State University of New York Press, 2004.

${ }^{14}$ A. Gnisci, Mondializzare la mente, Roma, Cosmo lannone, 2006; Idem (a cura di), Forse questo è il confine, (in collaborazione con F. Bettini), Roma, Meltemi, 1999; Idem, Creolizzare I'Europa. Letteratura e migrazione, ivi, 2003; Idem, Poetiche dei mondi, Roma, Meltemi, 1999; A. Gnisci, F. Sinopoli, N. Moll, La letteratura del mondo nel XXI secolo, Milano, Bruno Mondadori, 2010.

${ }^{15}$ P. Khouma, lo, venditore di elefanti, a cura di O. Pivetta, Milano, Baldini Castoldi, 2006 [1990]; S. Methnani/M. Fortunato, Immigrato, Milano, Bompiani, 2006 [1990]; M. Bouchane, Chiamatemi Alì, a cura di C. de Girolamo e D. Miccione, Milano, Leonardo, 1990.

${ }^{16}$ D. Comberiati, Scrivere nella lingua dell'altro. La letteratura degli immigrati in Italia (1989-2007), Bruxelles, Peter Lang, 2010, p. 15.

${ }_{17}$ Cfr. ivi, p. 27 ss.

${ }^{18}$ T. Ben Jelloun, Dove Io Stato non c'è. Racconti italiani, Torino, Einaudi, 1991. Per un'analisi del racconto di Ben Jelloun cfr. M. Kleinhans, “"Dolce stil nero" - Neue Wege der afroitalienischen Migrationsliteratur', in: Zibaldone. Zeitschrift für italienische Kultur der Gegenwart, 52 (autunno 2011), p. 77 ss.
} 
razzismo. In ogni caso risulta la necessità di una terapia, un bisogno di scrivere per canalizzare le paure e le angosce, e per gridare fuori l'ansia, l'ingiustizia e il dolore.

Nella letteratura postcoloniale l'aspetto della violenza proviene sia dall'atto coloniale in sé, sia dal parametro transculturale che confronta il soggetto migrante con conflitti psicologici e identitari, di ambivalenza, non-appartenenza e ibridità, ma anche culturali, relativi ai conflitti di integrazione e assimilazione sociale, religiosa o geografica. Da questo punto di vista risulta logico che moltissime opere prime degli autori italofoni qui in questione siano contrassegnate da un autobiografismo che non è da intendere necessariamente come manifestazione del subgenere classificatorio dell'autobiografia secondo la definizione proposta da Philippe Lejeune ( $\mathrm{e}$ est un autre. L'autobiographie de la littérature aux médias, 1980), ma come un prodotto artisticoletterario che risulta da fenomeni post e transmoderni come una categoria estetica caratterizzata dalla 'presenza generica del soggetto' in molti testi di diversa provenienza, insomma dal sapore testimoniale nuovo, come spesso dimostrano anche le reali biografie transculturali di questi scrittori. ${ }^{19}$

L'autobiografia 'selfhelpista' spesso viene giudicata come un testo il cui 'tenore letterario risulta assai basso'. ${ }^{20}$ Se rivolgiamo la nostra attenzione alla letteratura di migrazione in generale però, possiamo constatare che a partire dall'inizio della modernità fino ai giorni nostri la letteratura autobiografica cerca di raccontare il mondo catastrofico nei termini di un racconto di vita, ${ }^{21}$ e che la nuova letteratura italofona non $\mathrm{fa}$ altro che reagire a questa tradizione rinnovandola e proponendo nuove forme e riflessioni acute. Tale genere cerca di decostruire l'io empatico della tradizione umanistica frammentandolo, illuminandolo in modo pluriforme e legandolo alla categoria dello spazio. Ne risulta una comprensione performativa e una dislocazione del racconto di vita che nella letteratura degli immigrati italofoni sperimenta una rinascita ottenendo un ringiovanimento letterario inaspettatamente positivo. Lo è tanto più se si paragona il caso italiano alle situazioni nei paesi vicini - la Francia, la Svizzera, la Germania e l'Inghilterra. Infatti la svolta da paese di emigrazione a paese di immigrazione di massa in Italia è avvenuta solo nel corso degli ultimi trent'anni e quindi con un grande ritardo. ${ }^{22}$ Ciò nonostante la letteratura italofona può vantarsi di un'attività produttiva assai ricca, notevole e significativa in termini quantitativi e discorsivi.

\footnotetext{
${ }^{19}$ Pur non definendo in modo del tutto soddisfacente e completo il nuovo genere che è la letteratura della migrazione qui in discussione, il termine dell'autobiografismo può essere utile per ricordare l'elemento soggettivo, personale e psicologico che la letteratura della migrazione utilizza. Con autobiografismo intenderemo allora non autobiografia in sensu strictu, ma la percepibile e comprovata presenza dell'io lirico o narrativo che lo scrittore adopera per modellare il suo testo sul piano estetico: "Autobiografismo è la presenza generica del soggetto nella propria opera letteraria. In questo senso qualsivoglia genere letterario può essere pervaso di autobiografismo perché l'autore può parlare di se stesso perfino nei generi più impersonali e oggettivi', A. Battistini, Autobiografismo versus Autobiografia, http: / / solettere.altervista.org/index2.php?option=com_sobi2\&sobi2Task=dd_download\&fid=11\&format=html (15 aprile 2012), p. 1.

20 Ivi, p. 12.

${ }^{21}$ P. Braun e B. Stiegler (a cura di), Literatur als Lebensgeschichte. Biographisches Erzählen von der Moderne bis zur Gegenwart, Bielefeld, Transcript, 2012.

${ }^{22}$ Cfr. B. Le Gouez, 'Ist “Multikulti” eigentlich gescheitert? Italiens Afrikaner und die Interkulturalität', in: Zibaldone. Zeitschrift für italienische Kultur der Gegenwart, 52 (autunno 2011), p. 59.
} 


\section{La questione gender}

In effetti, il fenomeno dell'immigrazione in Italia ha creato nuovi gruppi subalterni all'interno della società: si pensi non solo agli africani - che hanno inaugurato a livello letterario il nuovo Dolce stil nero ${ }^{23}$ - e agli albanesi - inizialmente percepiti ex negativo come mostra il giornalista Gian Antonio Stella in L'orda. Quando gli albanesi eravamo noi $(2002)^{24}$ - ma soprattutto anche ai 'terroni' emigrati all'interno dell'Italia ovvero agli scrittori meridionali spesso sottovalutati nel loro impeto critico, transculturale e/o pacifista - pensiamo per esempio allo scrittore siciliano Giuseppe Bonaviri (1924-2009) come 'padre' della scrittura transculturale postmoderna $-{ }^{25}$ agli autori arabi assimilati come Magdi Allam (lo amo I'Italia, ma gli italiani la amano?, 2007) ${ }^{26}$ e alle donne scrittrici.

Focalizzando la questione gender e analizzando la sfera letteraria italofona legata al potere coloniale secondo lo stato della ricerca attuale, osserviamo che inizialmente il ruolo delle donne ha luogo nelle figure femminili rappresentate nei romanzi coloniali di autori maschili. Il sogno coloniale dell'Italia, che include il cosiddetto sogno africano, nel Dopoguerra ha influenzato i due noti romanzi sul colonialismo italiano in prospettiva maschile: Ennio Flaiano illustra in Tempo d'uccidere (1947) la campagna militare fascista in Etiopia, mentre l'autore toscano Mario Tobino ci narra quella in Libia (II deserto della Libia, 1952). ${ }^{27}$ Ambedue gli scrittori vi elaborano le loro esperienze autobiografiche durante le guerre italiane in Africa: il pescarese aveva combattuto nel novembre 1935 nella guerra contro l'Etiopia, Tobino invece a partire dal 1940 era stato di leva in Libia per quasi due anni. Nel romanzo di Tobino, dal punto di vista diegetico, il Dottor Marcello del trentunesimo corpo ufficiali della sanità dell'esercito italiano, arrivato a Tripoli nel 1940, si vede confrontato con le donne arabe che evocano nel protagonista immagini stereotipate legate alle Mille e una notte, raccolta di fiabe d'origine persiana che, sia detto fra parentesi, storicamente in Italia era conosciuta già a partire dal Trecento e vi circolò già molto prima che nel resto dell'Europa, che la scoprì solo attraverso la traduzione in francese nel Settecento. Tobino utilizza quindi elementi di un'alterità che da secoli è assimilata in Italia essendo entrata nella memoria culturale collettiva del paese e usando, in altre parole, un materiale letterario genuinamente transculturale. Flaiano, invece, in Tempo d'uccidere ci propone un giovane soldato che incontra nel bosco una donna etiope che uccide per sbaglio dopo

\footnotetext{
${ }^{23}$ Cfr. Kleinhans, “"Dolce stil nero”', cit.

${ }^{24}$ Dopo il successo del libro è stato realizzato un ononimo spettacolo teatrale sulla emigrazione italiana nelle Meriche con materiale storico e documentario registrato su dvd: G. A. Stella, Il viaggio più lungo. L'odissea dei migranti italiani, musiche di G. Bertelli e della Compagnia delle Acque, Milano, Rizzoli, 2010 (la pubblicazione consiste di: 'Lo spettacolo' [dvd]; 'Dizionario essenziale' [libro]).

${ }^{25}$ Cfr. D. Reichardt, 'Bonaviri terapeuta. Letteratura di migrazione e scrittura empatica', in: A. Pagliardini e A. Vranceanu (a cura di), Migrazione e patologie dell'humanitas nella letteratura europea contemporanea, Frankfurt a.M., Peter Lang, 2012, pp. 219-230. In questo saggio ho evidenziato alcune caratteristiche comuni degli scrittori di migrazione, analizzando la transculturalità dell'autore siciliano (idem, pp. 222-225) e il ruolo di Bonaviri 'da pioniere, precursore o da "padre" della letteratura di migrazione' (p. 225).

${ }^{26}$ Per un'analisi comparatista del testo di Allam che dal punto di vista culturale è da considerare un caso profondamente ambivalente cfr. D. Reichardt, 'Cultura cosmopolita: migrazione, modificazione e modernità liquida nei testi di Salah Methnani, Magdi Allam e Cristina Ali Farah', in: C. Salvadori Lonergan et al. (a cura di), Insularità e cultura mediterranea nella lingua e nella letteratura italiana. Atti del XIX Congresso dell'A.I.P.I. Cagliari, 25-28 agosto 2010, vol. 2 L'altro Mediterraneo e altre sponde, Firenze, Franco Cesati, 2012, pp. 401-404.

${ }^{27}$ Fra i precursori di questi due romanzi ricordiamo A. Boito (L'alfier nero, 1876), E. Salgari (La favorita del Mahdi, 1887; Sull'Atlante, 1888; I predoni del Sahara, 1903) o R. Bacchelli (Mal d'Africa, 1934).
} 
averla corteggiata ed è tormentato da forti sensi di colpa. Il romanzo finisce in modo aporetico; rimangono i ricordi e i dubbi della guerra. Sessant'anni più tardi l'autrice di origine etiope Gabriella Ghermandi riscriverà in Regina di fiori e di perle (2007) questa scena chiave invertendo la relazione fra uomo e donna. ${ }^{28}$

Approfondendo ancora la cronologia delle pubblicazioni possiamo verificare che prima di Flaiano e Tobino si era già espressa artisticamente anche una donna sull'esperienza colonialista: Pina Ballario scrisse il romanzo sull'Eritrea La sposa bianca (1934), ${ }^{29}$ quasi un'anticipazione della tesi di Albert Memmi, che nel suo Portrait du colonisé précédé du Portrait du colonisateur (1966) si era riallacciato alla dialettica padrone-servo hegeliana (Herr-Knecht-Dialektik), precisando poi nel suo libro intitolato La dépendance (1979) che alla fine del Novecento saremmo diventati testimoni di due avvenimenti significativi: la fine della colonizzazione e l'abrogazione della repressione femminile. ${ }^{30}$

Dalle costanti dell'autobiografismo e della violenza nella letteratura italofona postcoloniale emergono, sulle soglie del Duemila, il motivo della violenza nel contesto globalizzato e la letteratura programmaticamente transculturale in Italia. ${ }^{31}$ La violenza ora non è più un mero fenomeno di guerra, costituita da sottomissione coloniale e brutalità fisica, ma diventa più sottile, celata dietro il velo della convivenza sociale, tendenzialmente più astratta e psicologica. La tematica identitaria è nettamente al centro dell'attenzione. Essa viene interpretata in termini biografici e culturali soprattutto nelle opere delle scrittrici migranti. ${ }^{32}$ Qui s'incrociano in maniera particolarmente felice i tradizionali parametri della scrittura femminile: la scrittura di viaggio, il diario, il romanzo di famiglia, l'autobiografia.

Continuando l'itinerario del romanzo e focalizzandoci ancora sulla letteratura postcoloniale, a partire dal 1990 si forma un gruppo di autrici che provengono dalle ex colonie italiane. Questo gruppo usa la lingua italiana, secondo Jennifer Burns, ${ }^{33}$ motivato da 'un desiderio d'inclusione nel paese d'adozione [...] non considerando l'italiano una lingua-simbolo di dominazione, quanto piuttosto una lingua neutra'. ${ }^{34}$ Qui spiccano i romanzi di Erminia Dell'Oro dall'Eritrea (Asmara addio, 1988), Ribka Sibhatu (Aulò. Canto-poesia dall'Eritrea, 1993) che è tra le prime scrittrici, assieme a Shirin Ramzanali Fazel e Nassera Chohra, a scrivere un libro senza l'aiuto di un co-autore, Cristina Ali Farah di origine somala (Madre piccola, 2007), Gabriella Ghermandi dall'Etiopia (Regina

\footnotetext{
${ }^{28}$ Cfr. S. Zangrando, 'Literatur und Migration. Zu einigen afroitalienischen Autoren', in: Zibaldone. Zeitschrift für italienische Kultur der Gegenwart, 52 (autunno 2011), p. 104.

${ }^{29}$ Per un'analisi più dettagliata del romanzo cfr. R. Pickering-Lazzi, 'Mass-mediated fantasies of feminine conquest', in: P. Palumbo (a cura di), A place in the sun. Africa in Italian colonial culture from postunification to the present, Berkley, University of California Press, 2003, p. 212 ss.

${ }^{30}$ Cfr. A. Memmi, 'Nachwort zur deutschen Ausgabe von 1980', in: idem, Der Kolonisator und der Kolonisierte. Zwei Portraits, Hamburg, Europäische Verlagsanstalt, 1994, p. 133.

${ }^{31}$ Per meglio definire la nozione della letteratura transculturale e l'effetto estetico transculturale voluto e quindi programmato da parte degli autori della migrazione cfr. Reichardt, 'Cultura cosmopolita', cit.; o anche: S. Brancato, 'Transculturalità e transculturalismo: i nuovi orrizonti dell'identità culturale', ALL L’Associazione, 2004, http://all.uniud.it/all/simp/num2/articoli/art4.html (16 aprile 2012).

${ }^{32} \mathrm{Cfr}$. il capitolo su 'La produzione letteraria delle scrittrici migranti' in: Comberiati, Scrivere nella lingua dell'altro, cit., pp. 75-102.

${ }^{33}$ Cfr. J. Burns, 'Language and its alternatives in Italophone migrant writing', in: J. Andall e D. Duncan (a cura di), National belongings. Hybridity in Italian colonial and postcolonial cultures, Bern, Peter Lang, 2010, pp. 127-147.

${ }^{34}$ La citazione è presa dalla recensione del saggio inglese di J. Burns, scritta e pubblicata da C. De Santi in lingua italiana, in: Annali d'Italianistica, 29 (2011), p. 478.
} 
di fiori e di perle, 2007) e Igiaba Scego di origine italo-somala (La mia casa è dove sono, 2010), figura paradigmatica della nuova 'cittadina' transculturale italiana. ${ }^{35}$

Genuinamente transculturali sono anche i romanzi di Fatima Ahmed (Aukuí, 2008), nata in Cambogia da padre somalo e madre indo-vietnamita, e appunto Shirin Ramzanali Fazel (Nuvole sull'Equatore. Gli italiani dimenticati. Una storia, 2010), nata a Mogadiscio da padre pakistano e madre somala. La forte presenza delle scrittrici italofone ${ }^{36}$ si riflette tra l'altro nelle pubblicazioni del concorso letterario nazionale Lingua Madre, progetto piemontese riservato alle donne straniere che utilizzano l'italiano 'ridisegnando la mappa culturale del nuovo millennio' ${ }^{37}$ e le relazioni con le altre donne, l'ultimo volume della collana essendo apparso nel 2010 ormai in quinta edizione.

Nelle opere di Sibhatu, Scego, Ali Farah e Ghermandi traspaiono sia la violenza nell'ottica femminile, sia le esperienze biografiche. Mentre Ribka Sibhatu ha alle spalle un'incarcerazione subita in Eritrea, Igiaba Scego soffre del razzismo che la fa sentire straniera in Italia, paese dove è nata. Nell'opera di Cristina Ali Farah la guerra civile in Somalia segna un'impronta traumatica, mentre Gabriella Ghermandi elabora nei suoi testi il passato coloniale dell'Etiopia. Nelle opere di queste autrici l'oralità serve da principio unificante. Già il titolo di Aulò. Canto-poesia dell'Eritrea, Sibhatu richiama l'oralità come elemento centrale del proprio bagaglio culturale, mentre nei romanzi La mia casa è dove sono di Scego e Regina di fiori e di perle di Ghermandi prevale la tematica di narrare anche testualmente delle storie inizialmente concepite per la trasmissione orale. Ali Farah infine inserisce in diverse situazioni narrative di Madre piccola un'intervista, una telefonata o vari altri dialoghi sottolineando il 'parlato' ed il carattere polifono del testo. I parametri del trauma e dell'oralità sono costanti nelle opere di tutte queste donne scrittrici.

\section{L'effetto terapeutico}

Dal punto di vista dei Subaltern Studies ${ }^{38}$ lo sviluppo della letteratura della migrazione italofona è senz'altro un elemento positivo, specialmente perché il fenomeno artisticoletterario dopo un ventennio di modesta attenzione da parte della critica e grazie anche a un lavoro attento e sensibile di alcune case editrici e dei media, ora sta subentrando anche visibilmente nella ricezione accademica. In rapporto alla scrittura femminile nelle nostre società di stampo europeo-occidentali, è certamente ancora troppo presto per poter parlare di una rivoluzione simbolica avvenuta nel senso di una trasformazione radicale come la rivendica Pierre Bourdieu (La domination masculine, 1998), perché le donne dominate possano prendere una posizione davanti agli uomini dominatori e davanti a loro stesse che sia identica a quella del dominatore.

Possiamo però sperare insieme a Sandra Ponzanesi ${ }^{39}$ in maniera forse già più concreta che la letteratura postcoloniale e migrante italofona - e in special modo quella

\footnotetext{
${ }^{35}$ Ulteriormente in questo contesto si è parlato anche dei nuovi italiani ovvero di 'una nuova specie d'italiani' (J. Mabiola Gangbo, Verso la notte Bakonga, Portofranco, Lupetti e Fabiani, 1999, p. 135).

${ }^{36}$ Dei 86 scrittori presentati da Comberiati nell'appendice del suo saggio (Comberiati, Scrivere nella lingua dell'altro, cit., pp. 261-282) ben 39 sono di sesso femminile.

${ }^{37}$ La citazione è presa dal testo della bandella posteriore del volume di D. Finocchi (a cura di), Lingua Madre. Duemiladieci. Racconti di donne straniere in Italia, Torino, Edizioni SEB 27, 2010.

${ }^{38}$ Per una definizione di questo tipo di ricerca cfr. l'introduzione di V. Chaturvedi nell'antologia Mapping subaltern studies and the postcolonial (a cura di idem, London/New York, Verso, 2000, p. VIII ss.).

${ }^{39}$ Ponzanesi scrive nella edizione del 1999 (Paradoxes of postcolonial culture. Contemporary women writing of the Indian and Afro-Italian diaspora, Enschede, Print Partners Ipskamp, 1999, p. 330): 'Very likely in the
} 
femminile - venga riconosciuta non solo per le tematiche varie che ha contribuito al canone italiano, ma anche per $\mathrm{i}$ risultati estetici e letterari finora presentati. $\mathrm{Ci}$ aspettano indubbiamente ancora molti studi monografici e analisi specifiche per valutare meglio questo nuovo campo di ricerca, ma è evidente che da quando Angelo Del Boca nel 1965 (La guerra d'Abissinia 1935-1941) cominciò a rivelare le atrocità nascoste dietro l'immagine degli Italiani, brava gente? (2005), soprattutto il lavoro di Armando Gnisci ha cambiato lo sguardo sulle scritture postcoloniali e migranti. Il loro percorso artistico è inizialmente contrassegnato da uno stretto approccio autobiografico e da un impegno inter- e/o transculturale che mira a un discorso artistico che non solo sta rinnovando la letteratura italiana, ma che implica anche un'autentica rielaborazione del passato e una discussione critica sull'aspetto traumatico del vissuto.

Constatando che i testi degli scrittori migranti riflettono la subalternità come specifica forma di un'aggressione contro la loro stessa incolumità sociale, fisica e mentale, possiamo verificare con Dacia Maraini che la scrittura sia da valutare come malattia e terapia allo stesso tempo, come processo del sapere e della cura. ${ }^{40}$ Così anche nelle opere delle scrittrici che culturalmente provengono dalle ex colonie italiane, lo spazio della scrittura diventa un luogo di scontro e di incontro nel senso che ha recentemente illustrato Giulia Po, analizzando i romanzi di Clara Sereni: ${ }^{41}$ la scrittura, a questo punto, diventa un efficace strumento di sensibilizzazione verso questioni sociali come la diversità etnica o l'identità di genere.

Possiamo allora affermare che le quattro autrici italofone Sibhatu, Scego, Ali Farah e Ghermandi, che qui abbiamo presentate come casi letterari paradigmatici, ricorrono al parametro orale per restare connesse con la loro cultura d'origine da un lato, e per estetizzare, dall'altro lato, le loro ambivalenze canalizzando le sofferenze e amalgamando le culture. Cercando di compensare la loro doppia subalternità nel senso inteso dalla Spivak, strumentalizzando l'autobiografismo per un fine ultimo terapeutico e, allo stesso tempo, estetico, ed integrando nei loro testi il pensiero migratorio, postcoloniale e transculturale, questa letteratura emergente produce degli effetti di rappacificazione, di legame, di comprensione e solidarietà (femminile), propagando un umanismo polifonico comunicativo. Come la protagonista di Mille e una notte, la narratrice Sherazad, che racconta per sopravvivere, queste autrici del terzo millennio propongono con le loro opere sia un writing e talking back dalla periferia della società italiana, sia una nuova scrittura femminile italofona che riflette la cultura cosmopolita di donne migranti e la versione italofona di una world literature transculturale che riconnette l'Italia con il suo passato coloniale e con la globalizzazione.

future, if the multiculturalization of Italy proceeds at the speed of the last decade, there will be also a proper "post-colonial tradition" worthy of comparison with that of the English and French, not only with regard to the commonalities of issues dealt with, as already emphasized in this study, but also for aesthetic and literary achievements'.

${ }^{40}$ Questo pensiero viene espresso sia nella raccolta Amata scrittura. Laboratorio di analisi letture proposte conversazioni (a cura di V. Rosi e M. P. Simonetti, Milano, Rizzoli, 2008, p. 6, 59, p. 90, p. 96) di Dacia Maraini, sia nel suo recente volume La seduzione dell'altrove (Milano, Rizzoli, 2010, p. 15-18, p. 30 ss.).

${ }^{41} \mathrm{G}$. Po, Scrivere la diversità: autobiografia e politica in Clara Sereni, Firenze, Franco Cesati, 2012. 


\section{Parole chiave}

Autobiografia, scrittura femminile, subalterno, transculturalità, violenza

Dagmar Reichardt insegna Letteratura e Cultura Italiana ed Europea presso l'Università di Groninga. È membro del comitato direttivo dell'A.I.P.I. (Associazione Internazionale Professori d'Italiano) e della Società Austriaco-Canadese (Österreichisch-Kanadische Gesellschaft). È autrice di numerose pubblicazioni nell'editoria tedesca e di saggi accademici su temi dell'italianistica. Dopo lo studio di filologia romanza all'Università di Amburgo dove ha conseguito il dottorato di ricerca sull'autore Giuseppe Bonaviri, ha insegnato presso le Università di Brema e di Innsbruck. Si è specializzata sulla letteratura siciliana, sul Novecento italiano e su argomenti inerenti agli Studi Culturali, Transculturali e Postcoloniali e alla Letteratura Comparata.

Dept. of European Languages and Cultures ETC

Faculty of Arts, University of Groningen

P.O. Box 716

9700 AS Groningen (The Netherlands)

d.h.a.reichardt@rug.nl

\section{SUMMARY}

\section{Subaltern Presence in Italy and Writing as Therapy}

From a theoretical and social point of view, modern Italophone postcolonial writing relegates to ways of receiving reality in the tradition of Verism and Neorealism, especially if aspects of violence are being considered. Notably the texts of female immigrant writers represent the subaltern (Gayatri C. Spivak) as they reflect the biographical traumatic experiences of their authors or historical violent backgrounds of the plots. In this sense, according to the paradigm of James W. Pennebaker (Writing Therapy), writing often assumes a therapeutical function. This article recalls the first attempts of articulating the Italian colonial past in literature, starting with Ennio Flaiano and Mario Tobino, and then focusing on the innovative migratory texts of female Italophone writers from the 1990s onwards, namely those of Ribka Sibhatu, Cristina Ali Farah, Gabriella Ghermandi and Igiaba Scego who culturally speaking all originate from former Italian colonies in Africa. By making use of the theorems of Armando Gnisci and Dacia Maraini, it becomes evident how female Italophone migrant writing must be recognized not only as a process of sensitization, but also as illness and therapy at the same time. Thus, the act of writing creates a transcultural space of both conflict and encounter, in which a talking back from the periphery of Italian society takes place, with the effect of reconciling Italy with its colonial history and globalized present. 